\title{
Mitral annuloplasty detachment secondary to unnoticed unruptured left sinus Valsalva aneurysm
}

\author{
Yolanda Carrascal ${ }^{1}$, Gregorio Laguna ${ }^{1}$, Ana Revilla ${ }^{2}$, Miriam Blanco ${ }^{1}$ \\ ${ }^{1}$ Cardiac Surgery, University Hospital Valladolid, Spain \\ ${ }^{2}$ Cardiology Department, University Hospital Valladolid, Spain
}

\begin{abstract}
Symptomatic unruptured Valsalva sinus aneurysms (VSA) can manifest as progressive aortic regurgitation, angina, heart failure, thrombotic embolization or arrhythmias. Presented herein, is a 41-year-old patient with Gray Platelet Syndrome (GPS) and mitral regurgitation known since childhood. Two years prior, he had presented with symptomatic severe mitral valve regurgitation, repaired by posterior leaflet quadrangular resection and flexible annuloplasty ring. At 3-month follow-up, echocardiography showed an eccentric mild-to-moderate regurgitant jet from the anterior commissure of the mitral valve. Eleven months later, a previously undiagnosed image, corresponding to unruptured asymptomatic left VSA (Fig. 1A, Suppl. Video 1) as well as left coronary cusp prolapse, was identified. Mitral regurgitation increased to moderate due to anterolateral partial detachment of annuloplasty ring (Fig. 1B). Computed tomography confirmed a left VSA, which extended into
\end{abstract}

interventricular septum and anterior commissure of the mitral valve (Fig. 1C).

Only 1-6\% of VSA affect left sinus, exceptionally extend into interventricular septum. In this case, tissue weakness favored by protruding left VSA, appeared directly related to progressive partial annuloplasty ring detachment (Fig. 1D-G). Probably, the flexible annuloplasty ring allowed a more natural movement of the valve during the cardiac cycle, avoiding early significative mitral regurgitation after the first surgery. Closure of VSA with bovine pericardial patch and resuture of annuloplasty ring dehiscence finally corrected left aortic cusp prolapse as well as mitral regurgitation (Fig. 1H, I). Although VSA is a rare condition, a detailed and complete preoperative echocardiographic examination (not only referred to known mitral disease) would have prevented further surgery in a patient with a high-risk hematologic disease.

Conflict of interest: None declared

Address for correspondence: Dr. Yolanda Carrascal, MD, PhD, Cardiac Surgery Department, University Hospital, Avda. Ramón y Cajal-3, 47005 Valladolid, Spain, tel/fax: 0034983420000, e-mail: ycarrascal@hotmail.com 


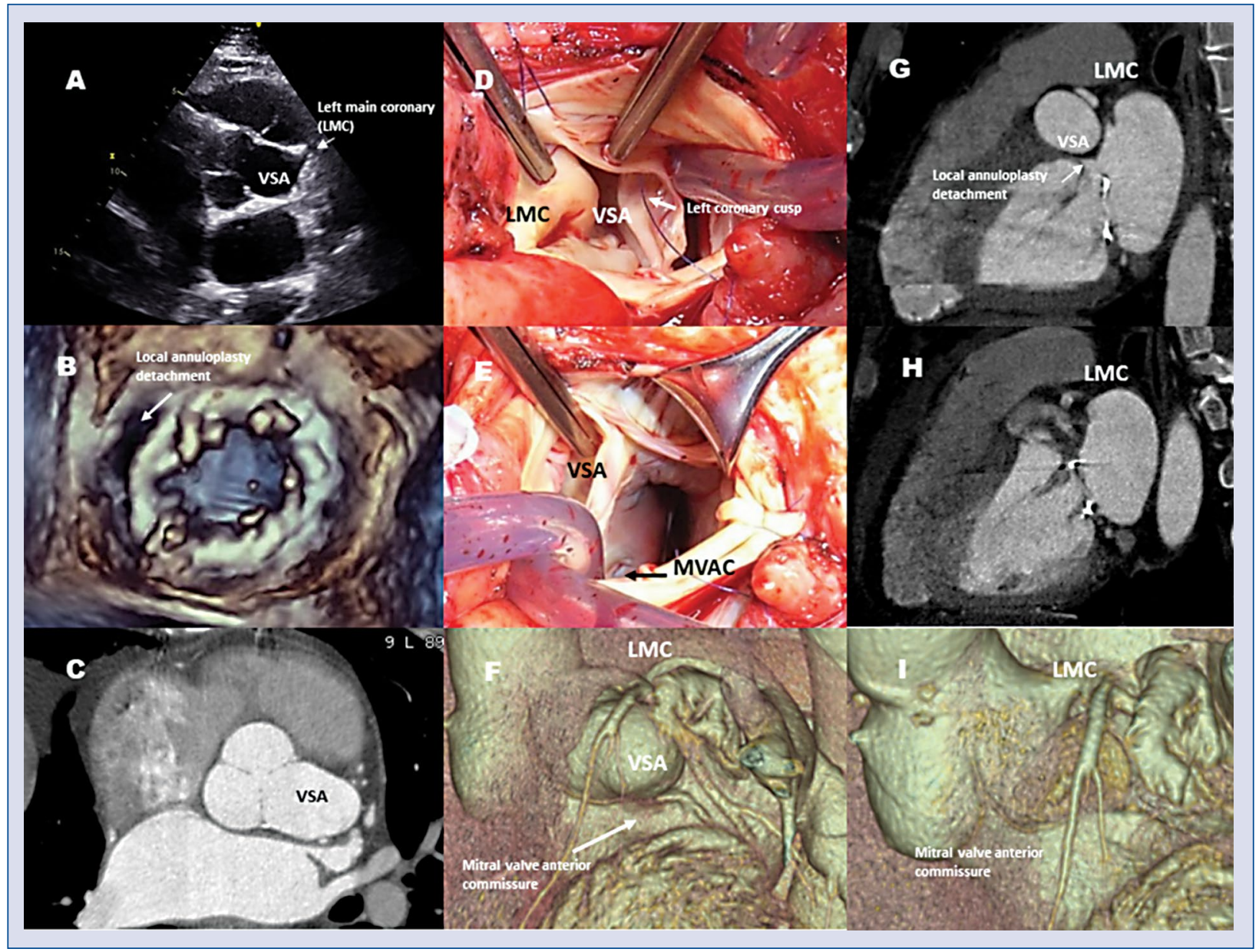

Figure 1. Preoperative echocardiography (A); magnetic resonance imaging $(2.8 \times 3 \mathrm{~cm})(\mathbf{C}, \mathbf{F}, \mathbf{G})$ and intraoperative (D, E) view of left aneurysm sinus of Valsalva, local annuloplasty detachment (white arrow) (B, G) and postoperative magnetic resonance imaging $(\mathbf{H}, \mathbf{I})$; LMC — left main coronary; MVAC - mitral valve anterior commissure; VSA — Valsalva sinus aneurysm. 